Chapter 8

\title{
Praziquantel and Arachidonic Acid Combination - An Innovative Approach to the Treatment of Schistosomiasis
}

\author{
Hatem Tallima, Kevin Hadley and Rashika El Ridi \\ Additional information is available at the end of the chapter \\ http://dx.doi.org/10.5772/61185
}

\begin{abstract}
Schistosomiasis is a debilitating disease caused by trematode worms of the genus Schistosoma. Three members Schistosoma mansoni, Schistosoma haematobium, and Schistosoma japonicum are responsible for the great majority of human infections. Schistosomiasis is widespread in sub-Saharan Africa, several countries of the Middle East, South America, and South-East Asia. Vaccination against the infection would be the most reliable way to combat the infection and decrease or interrupt its transmission, but a commercial vaccine is still unavailable. Praziquantel (PZQ) is the only drug considered for schistosomiasis treatment as it is effective against the major human schistosomes, commercially available, cost-affordable, and elicits limited side-effects. Several reports documented the highly significant PZQ efficacy in treatment of light infections in areas of low S. mansoni and S. haematobium endemicity and PZQ use. Chemotherapy with PZQ alone of patients residing in regions of high schistosome endemicity and afflicted with light, moderate, or heavy infection is not efficacious. Accordingly, we propose implementation of cost-affordable arachidonic acid (ARA), a polyunsaturated omega- 6 fatty acid and efficacious in vitro and in vivo schistosomicide, for oral therapy of children with Schistosoma mansoni and Schistosoma haematobium light infection, as adjunct to PZQ for cure of children with moderate and heavy infections, and for counteracting schistosome resistance to PZQ that arises in endemic areas exposed to repeated and intense PZQ mass treatment campaigns.
\end{abstract}

Keywords: Praziquantel, Arachidonic acid, Schistosomiasis, Chemotherapy, Combination chemotherapy 


\section{Introduction}

Schistosomiasis is a debilitating disease caused by dioecious (having separate sex) trematode worms of the genus Schistosoma. Three members, Schistosoma mansoni, Schistosoma haematobium, and Schistosoma japonicum, are responsible for the great majority of human infections. Schistosomiasis is also called snail fever as the schistosomes' life cycle comprises asexual reproduction in an appropriate, fresh water snail. Actually, it is the presence of the specific snail vector that determines the prevalence of the disease, as infection is exclusively caused by the larvae (termed cercariae) shed by infected snails. The cercariae of S. mansoni, S. haematobi$u m$, and S. japonicum swim in fresh water and soon die if they fail to land on the skin of humans or other suitable mammalian hosts. The cercariae then penetrate the epidermis, linger there for one (S. japonicum) to three (S. mansoni, S. haematobium) days or more until extensive biochemical changes end by setting a protective, outer double-lipid bilayer armor. The larvae, now termed schistosomula, exit to the dermis and then make their way into dermal venous capillaries, en route to the lung and then liver. There the parasites start to feed and rapidly grow and mature, and the male carrying the female into its ventral gynecophoric groove or "schist" migrate to permanent residence in the inferior mesenteric (S. mansoni, S. japonicum) or the peri-vesical (S. haematobium) venous plexus. Until then, the worms are nearly innocuous causing very limited harm, especially in endemic regions. With the onset of female parasite egg deposition, harm starts and progresses into overt diseases [1-5].

To continue the life cycle, the ova must egress the mammalian host vasculature to the exterior to locate the intermediate snail host where asexual reproduction takes place. The parasite dwelling is nearest to the outlets of the lower colon and rectum via feces and the urinary bladder with urine. Eggs release proteolytic and other hydrolytic enzymes to transit the blood capillaries, and thereafter the large intestine (or urinary bladder) wall into the lumen. Besides releasing host matrix digestive enzymes, S. mansoni and S. haematobium additionally depend on the sharp, lateral and terminal spine, respectively. The massive daily egg migration, trapping, and calcification lead to edema, congestion, ulcers, lesions, petechial hemorrhage, necrosis, hyperplastic and hypertrophic changes, fibrosis, and excessive nodules and polyps formation in the intestine, symptoms collectively known as intestinal schistosomiasis [1, 2]. Continuous egg transit via the wall of the lower urinary tract to the lumen causes even more damage to the urinary tract and bladder, the severe symptoms of urinary schistosomiasis [3]. Beside the egg-induced overt mechanical injury and lesions, the presence of parasite molecules within the extracellular matrix signals danger and expectedly contributes to intense and prolonged generation of inflammatory mediators [4].

The "plat de resistance" is still away, as the most severe injury does not result from the eggs that escape to the exterior but from the eggs that fail to do so. Eggs trapped in the wall of the intestine or urinary tract drift and eventually accumulate in tissues of other organs, namely the liver, where they remain viable for approximately three weeks, and release soluble egg antigens (SEA) via their microscopic pores. The host responds to these insults through vigorous immunologic reactions. These intense immunological reactions are injurious to the host, not the egg that remains unscathed, until its viability becomes exhausted. Lymphocytes, eosinophils, basophils, and macrophages accumulate around the egg in attempt to prevent its contents from seeping and disseminating, thus forming large granulomas. The granulomas 
gradually begin to encompass the entire organ, progressing to fibrosis with excessive accumulation of collagen and extracellular matrix proteins, obstructive vascular lesions, vascular hypertension, neo angiogenesis, splenomegaly, esophageal varices, and signs of hepatocellular (S. mansoni) or kidney (S. haematobium) failure [1-3, 5].

The transiting eggs-induced mechanical injury and the immunological reactions to the entrapped eggs manifest into colitis, diarrhea, blood in stool or urine, abdominal discomfort and pain, urodynamic abnormalities, fatigue, lower exercise and work tolerance, impaired cognitive capacities, and retarded development. Chronic infection leads to organ dysfunction, intense vascular complications, and additionally, predisposes to other even more severe viral and bacterial infections, cancer development, and ultimately death [1-3,5].

Detection of eggs in stool specimens is the gold standard for diagnosis of the infection. In chronic infections, i.e., in adults residing in endemic areas, continuous egg deposition and migration via the wall of the lower intestine and the urinary bladder and tracts generate fibrosis in the submucosa and hypertrophy in the muscularis mucosa, and consequently, a barrier is raised to the usual route of ova transit from the surrounding veins to the lumen of the gut or urinary bladder $[2,3]$. Expectedly, the gold standard method of egg detection is entirely unreliable in chronic infections. Diagnostic methods based on serological detection of antibodies to the parasite antigens do not lack sensitivity, but lack specificity and additionally may not be used to monitor the outcome of therapy or to differentiate between present and past infection. Diagnostic methods based on antigen detection in serum, urine or stool, and saliva lack both sensitivity and specificity and together with the methods based on molecular biology advances are cumbersome, costly, and none has been adapted for routine screening in endemic regions, all situated in the developing world [5 and references therein, 6]. Epidemiological surveys are costly and require thorough scientific and political involvement, both often failing in developing countries. Based on the above, it may be foreseen that the figures on the prevalence of schistosomiasis, namely 252 million infected, are a gross underestimate. Yet, it is certain that the figures of 800 million persons, namely children, residing in rural areas are at risk of the infection, and yearly deaths as high as 200,000 are correct [6-9]. Schistosomiasis is widespread in sub-Saharan Africa, several countries of the Middle East, South America, and South-East Asia [6-10]. Vaccination against the infection would be the most reliable way to combat the infection and decrease or interrupt its transmission, but a commercial vaccine is still an unmet clinical need. Praziquantel is the only drug considered for schistosomiasis treatment as it is effective against the major human schistosomes, commercially available, costaffordable, and elicits limited side-effects [reviewed in 5, 11, 12].

\section{Chemotherapy}

\subsection{Praziquantel}

\subsubsection{Structure and schistosomicidal effects in vitro and in experimental animals}

Praziquantel (PZQ), $\mathrm{C}_{19} \mathrm{H}_{24} \mathrm{~N}_{2} \mathrm{O}_{2}$ (2-cyclohexylcarbonyl-4-oxo-1,2,3,6,7,11b-hexahydro-4Hpyrazino[2,1-a]iso-quinolin-4-one) (Figure 1) is a hydrophobic molecule of molecular weight 
$312.4 \mathrm{~g} /$ mole, for all this lengthy formula, smaller than the molecular weight of cholesterol $386.6 \mathrm{~g} / \mathrm{mole}$ ), which schistosomes cannot synthesize de novo and rely on absorption from the host via the tegument [13].

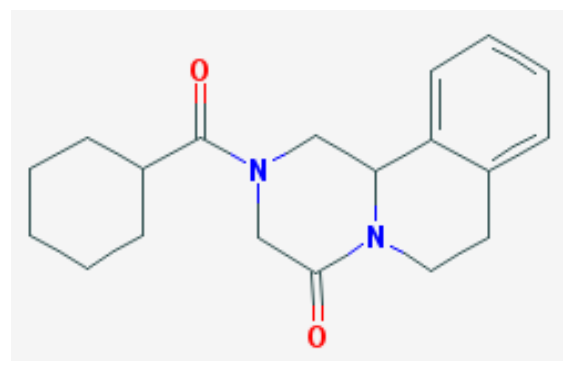

Figure 1. Structure of praziquantel.

Because of its small molecular weight and hydrophobic nature, PZQ may access and interact with the schistosome outer lipid bilayer, expectedly causing disruption, degeneration, and disintegration. Adult S. mansoni were exposed in vitro to 1, 10, or $100 \mu \mathrm{g} / \mathrm{ml} \mathrm{PZQ} \mathrm{for} \mathrm{5,} \mathrm{15,} \mathrm{30,}$ or 60 minutes, and examined by light and electron microscopy. Severe body contraction and complete cessation of movement were associated with the appearance of numerous small areas of vacuolization and vesicle formation distributed all over the parasite surface, their number increasing with PZQ concentration and exposure time and eliciting disruption of the apical tegumental layer $[14,15]$.

Mice orally treated with $500 \mathrm{mg} / \mathrm{kg}$ PZQ seven weeks following infection with S. mansoni showed dislodgment of the worms from the veins of the intestine to the portal circulation, and pronounced vacuolization of the tegument as early as one hour after treatment, indicating that in vitro and in vivo likewise, PZQ suddenly and abruptly affects the worm physiology. Soon after, neutrophils and eosinophils attached to the bleb-like vacuoles and succeeded in infiltrating the interior of the worms leading to progressive internal lysis and eventually total disintegration [15]. Yet, several reports indicated that 21- and 28-day worms are less sensitive to PZQ action in vivo [16, 17].

Lung-stage schistosomula were recovered one hour after treatment of 6-day S. mansoniinfected mice with 0 or $200 \mathrm{mg} / \mathrm{kg}$ PZQ, and examined by indirect membrane immunofluorescence using chronic infection serum. Larvae recovered from untreated mice were entirely negative, a finding we repeatedly confirmed decades later [11, 12 and references therein]. Larvae harvested from PZQ-treated hosts revealed exposure of hitherto hidden surface membrane molecules, likely consequent to interaction of PZQ with the lung-stage schistosomula apical lipid bilayer [18]. Proven PZQ-mediated exposure of larval and adult S. mansoni surface membrane antigens to hitherto barred antibody access suggested that PZQ in vivo action is due to the synergy between its direct effect on the worm and host-immune effectors, principally antibody-dependent cell- and complement-mediated cytotoxicity and granulocytes cytolytic action [19-22]. 
Praziquantel was shown to be equally effective against S. haematobium and S. japonicum in vitro and in vivo, inducing spastic paralysis, tegumental vacuolation and disruption, and surface membrane exposure to host antibodies and other immune effectors [23-25].

\subsubsection{Mechanism of action}

The pyrazino-isoquinoline ring system of PZQ represents a completely novel structure in antihelminthic chemotherapy, and additionally, its schistosomicidal mode of action is not intuitively clear [26]. The most immediate PZQ effect, at least in vitro, is dramatic parasite contraction and spastic paralysis, followed by outer membrane vacuolation, blebbing, and disruption. Thus, one hypothesis to explain PZQ mode of action is that the drug inserts itself in the membrane, destabilizes the double lipid bilayer [27-29], and subsequently interacts with muscle filaments. Due to its size and hydrophobic nature, PZQ likely intercalates within the phospholipid molecules of the worm apical lipid leaflets, undergoes hop diffusion in the plasma membrane, and permeates to access tegumental actin, myosin, and/or tropomyosin [27-30]. Adult worms' surface spines are crystalline structures consisting of filaments of actin that interact directly with the apical and basal membranes [31, 32]. Actin is present in the surface membrane tubercles and in the tegument [33]. More importantly, actin molecules are known to form a mesh or fence underneath surface plasma membranes [30, 34, 35]. Putative interaction of PZQ with actin in spines, tubercles, and tegument would affect its polymerization status, leading to surface membrane disruption. Pre-incubation of parasites in the presence $30 \mathrm{mM}$ magnesium before exposure to PZQ prevented contraction and tegumental disintegration in all worms [36]. This finding, coupled with the recent report showing magnesiumdependent modulation of myosin and actin functions, [37] may be construed to indicate that PZQ directly interacts with the parasite myosin and actin.

We have taken advantage of the Claisen Condensation Reaction to irreversibly bind the otherwise inert PZQ to cellulose acetate membrane and thus use affinity chromatography to isolate the parasite surface membrane molecule(s) that most selectively bind to PZQ. Amino acid microsequencing and immunogenicity studies indicated that PZQ preferably binds to schistosome actin at the exclusion of other adult worm surface membrane antigens. We have proposed that PZQ accesses actin of schistosomes and induces its polymerization, with subsequent parasite contraction and tegument disruption [38, 39]. Cytochalasin B disrupts actin polymerization by capping the fast-growing end of actin filaments. Pre-treatment of adult S. mansoni with cytochalasin B did not entirely prevent worm contraction and immobility, yet rendered the parasites refractory to killing by even exceedingly high PZQ concentrations. The results may easily be construed to indicate that PZQ-mediated actin polymerization is the major mechanism of PZQ killing [40]. Ramaswamy and colleagues expressed the cDNA library of $S$. mansoni on the surface of T7 bacteriophages and screened the displayed proteins with labeled PZQ. The results indicated PZQ binds to the N-terminal end of myosin light chain and to actin, and further revealed that $S$. mansoni myosin light chain is phosphorylated in vivo upon binding to PZQ [41].

The second major PZQ effect is abnormal calcium influx in the worm, leading to hypothesizing that voltage-gated calcium channel(s) on the surface of the worm is the elusive target of PZQ 
action [42-46]. However, Cioli and colleagues failed to detect the correlation between PZQinduced intra-worm calcium influx and parasite death [47]. In support, incubation of $S$. mansoni worms in the presence of seven different calcium channel blockers gave insignificant protection against PZQ lethal action except for nicardipine and nifedipine, which nevertheless did not prevent the spastic paralysis observed in worms observed in schistosomes exposed to PZQ, and allowed $50 \%$ of the worms to be killed by $3 \mu \mathrm{M}$ PZQ. In contrast, all worm survived exposure to $36 \mu \mathrm{M}$ PZQ following pre-treatment with the actin depolymerizing agent, cytochalasin B [40].

\subsubsection{Safety and efficacy}

Extensive use of PZQ for treatment and control of schistosomiasis requires a comprehensive understanding of efficacy and safety:

a. Of various doses for different Schistosoma species. PZQ is active against all schistosome species that infect humans. A multicenter, randomized, controlled trial of the efficacy and safety of single-dose PZQ at $40 \mathrm{mg} / \mathrm{kg}$ versus $60 \mathrm{mg} / \mathrm{kg}$ for treating intestinal schistosomiasis in the Philippines (S. japonicum), Mauritania, Tanzania, and Brazil (S. mansoni) enrolled 856 patients, aged 10-19 years and with $\geq 100$ eggs per gram of feces (epg). A total of $666(78 \%)$ reported adverse reactions, mostly abdominal pain, at 4 hours after administration with no highly significant differences between doses. Both doses were highly effective, as in Day 21 cure rates were $91.7 \%$ (86.6\%-98\% at individual sites) with $40 \mathrm{mg} / \mathrm{kg}$ and $92.8 \%(88 \%-97 \%)$ with $60 \mathrm{mg} / \mathrm{kg}$, suggesting there is no significant efficacy advantage of the 60 versus standard $40 \mathrm{mg} / \mathrm{kg}$ for therapy of intestinal schistosomiasis. Of note, there were no differences in efficacy outcomes with variations in transmission and infection intensities across the sites [48]. A meta-analysis of comparative and noncomparative clinical trials on PZQ clinical efficacy and tolerability for intestinal and urinary schistosomiasis, involved 19,500 patients, largely children, in 24 countries and 82 sites. Again, approximately $60 \%$ of the subjects receiving $40 \mathrm{mg} / \mathrm{kg}$ PZQ reported adverse reactions, namely abdominal pain. The WHO-recommended dose of PZQ $40 \mathrm{mg} / \mathrm{kg}$ achieved within 8 weeks cure rates of 94.7\% (92.2-98.0) for S. japonicum, 77.1\% (68.4-85.1) for S. haematobium, 76.7\% (71.9-81.2) for S. mansoni, and 63.5\% (48.2-77.0) for mixed S. haematobium/S. mansoni infections; the data revealed efficacy in some sites was significantly lower than expected [49].

b. For low, moderate, and heavy infections. In a study performed in 2009-2010, PZQmediated cure rates in 588 school-age children of S. mansoni endemic El Rouse village in Kafr El Sheikh at 4 weeks after a single PZQ dose of $40 \mathrm{mg} / \mathrm{kg}$ were $83 \%, 76 \%$, and $54 \%$ for light (<100 epg), moderate (100-400 epg), and heavy (>400 epg) infection, respectively, indicating that pre-treatment intensity of infection has a great influence on PZQ efficacy [50]. Decrease of cure rates with increasing pre-treatment egg counts was also observed among 611 S. mansoni-infected schoolchildren from three schools in northeast Ethiopia. Side-effects and symptoms, especially abdominal cramps, vomiting, diarrhea, and dizziness were reported by $91 \%$ of the children and were more pronounced in those with high pre-treatment egg excretion [51]. Among 253 S. mansoni-infected children in western 
Côte d'Ivoire treated with $60 \mathrm{mg} / \mathrm{kg} \mathrm{PZQ}$, there was a significant $(\mathrm{P}<0.01)$ association between cure rate and intensity of infection prior to treatment with highest cure rates observed in light infections [reviewed in 52]. Highest cure rates against $S$. haematobium infection among 675 primary schoolchildren in Zimbabwe were observed in those with light baseline infection; yet, a wide range of observed mild and transient side effects were not associated with egg intensity [53]. The remarkably low cure rates obtained from people with heavy S. mansoni or S. haematobium infections led King et al. [54] to advocate the repeat of PZQ treatment 2-8 weeks after the first dose. We have recently performed a study involving 260 S. mansoni-infected schoolchildren in Kafr El Sheikh, Egypt. Cure rates 6 weeks after a single dose of $40 \mathrm{mg} / \mathrm{kg}$ PZQ were $60 \%, 42 \%$, and $20 \%$ for light, moderate, and heavy infections, respectively [55], fully supporting the observation that PZQ efficacy greatly decreases with the increase of baseline infection levels.

c. In areas of low endemicity/limited PZQ treatment versus areas of high endemicity and massive, repeated PZQ administration. In our recent study involving 66 school-age children in low S. mansoni endemicity villages of Menoufiya, Egypt, PZQ cure rates at 4 weeks following treatment with a single dose of $40 \mathrm{mg} / \mathrm{kg}$ PZQ, were $87 \%$ and $83 \%$ for light and moderate infections, respectively, significantly higher than cure rates achieved in the high S. mansoni endemicity villages of Kafr El Sheikh [55, 56]. Our findings fully support the observations of King et al. [54] who reported that for subjects with S. mansoni or S. haematobium infection, cure rates were higher in communities having lower initial prevalence. Indeed, in Cameroon regions with low prevalence of schistosomiasis, and in Senbete Town, northeast of Ethiopia, treatment of schoolchildren with a single dose of 40 $\mathrm{mg} / \mathrm{kg}$ body weight resulted into cure rates of $80-95 \%$, with the majority of children reporting drug-related symptoms and malaise as late as 24 hours after treatment $[57,58]$. Conversely, a single oral dose at $40 \mathrm{mg} / \mathrm{kg}$ body weight induced only approximately $60 \%$ reduction in prevalence and epg in $<10-14$ year-old schoolchildren In Wondo Genet, southern Ethiopia, region with school age children prevalence rate of $75 \%$, i.e., high-risk communities. Approximately $83 \%$ of S. mansoni-infected children complained of headache, nausea, abdominal pain, bloody stool, vomiting, and fever at 24 hour post-treatment. These symptoms were associated with age $(P<0.001)$ and pre-treatment intensity of infection $(P<0.05)$ [59].

A study was performed along the high endemicity shores of Lake Victoria after 12.5 years of massive and repeated PZQ use involving 178 S. mansoni-infected men. The overall cure rate after a single PZQ dose was $66 \%$ ranging from $36 \%$ to $82 \%$. Of note, treatments administered in 2006 were significantly more likely to result in cure failures than treatments administered in 2004, the year in which PZQ efficacy was highest [60]. Similar observation was also reported for S. mansoni-infected schoolchildren in Kafr El Sheikh, Egypt. Thus, in a study performed in 2009-2010, PZQ-mediated cure rates in school-age children of S. mansoni high endemicity El Rouse village in Kafr El Sheikh were $83 \%, 76 \%$, and 54\% for light, moderate, and heavy infection, respectively, 4 weeks after a single PZQ dose of $40 \mathrm{mg} / \mathrm{kg}$ [50]. These cure rates were distinctly higher than those obtained in in the same areas at 6 weeks after PZQ therapy, suggesting reduction in PZQ efficacy 3 years later, following intense and repeated PZQ administration [55]. 


\subsubsection{Evidence for worm resistance to $P Z Q$}

Twenty years after its introduction, PZQ remains the drug of choice despite treatment failures and emergence of resistance being reported.

First indications on worm resistance to PZQ appeared in the Senegal River Basin, whereby 117 out of 130 selected subjects (87\%) were infected with S. mansoni with overall geometric mean epg of 478 . The overall cure rate 4 weeks after treatment with single standard PZQ dose was only $42 \%$. However, cure rate after a second round of treatment rose to $76 \%$, suggesting the low cure rate observed after one treatment was probably the result of a combination of high infection intensity and the maturation of pre-existing pre-patent S. mansoni infections [61]. Exceedingly low cure rates but similar conclusions were reached by Gryseels et al. [62], advocating there is no convincing evidence for emergence of PZQ-resistant S. mansoni in Senegal, and that the exceptionally low cure rates can be attributed to high initial worm loads and intense transmission in this area. However, low PZQ cures were again reported in children living in villages in Senegal River Basin with intense S. mansoni and S. haematobium transmission. Additionally, high prevalence and high infection intensity of S. mansoni and S. haematobium were still evident despite multiple rounds of chemotherapy, leading to the recommendation of new treatment regimens to control schistosomiasis in the school-age population [63].

Evidence for some degree of $S$. mansoni resistance to PZQ has been obtained using parasites taken from treated, but uncured human patients in Egypt and Senegal and in a laboratory isolate of $S$. mansoni subjected to successive passages under drug pressure, raising the need for alternative drugs to treat PZQ-resistant schistosomiasis [64-66]. Yet, there was no increase in drug failure, despite 10 years of therapeutic pressure in some Nile Delta villages where there had been resistant infections and worms with decreased response to PZQ [67]. In contrast to this optimistic note, a British traveler returning from East Africa failed to be cured despite several rounds of standard PZQ doses and no opportunity for re-infection [68]. In addition, there are several schistosomiasis cases caused by S. haematobium infections in which repeated standard treatment failed to clear the infection [69]. Isolates of $S$. mansoni obtained from patients from Kisumu, Kenya continuously exposed to infection as a consequence of their occupations as car washers or sand harvesters showed reduced PZQ susceptibility that was associated with previous PZQ treatment of the patient [70]. Recently, miracidia were hatched from eggs obtained from seven Kenyan car washers and used to infect snails. Shed cercariae were used to establish in mice a laboratory $S$. mansoni strain with significantly reduced PZQ sensitivity that was achieved within only 5 generations by administering increasing PZQ doses to the infected mice; the parasites were eventually able to withstand a normally lethal dose [71].

\subsubsection{Proposed mechanism(s) for PZQ resistance}

Resistance to a drug is defined as "genetically transmitted loss of sensitivity in a parasite population that was previously sensitive to the drug". Schistosomicidal action of oxamniquine (OXA) and hycanthone is dependent on activation by sulfotransferase enzymatic esterification and this ultimately results in the production of an electrophilic moiety capable of alkylating DNA $[72,73]$. Resistance of $S$. mansoni to OXA was attributed to loss-of-function mutations in 
a gene on chromosome 6 that was found to be encoding the enzyme, sulfotransferase [74]. Unlike OXA and hycanthone, there is no evidence PZQ interacts with schistosome DNA, inducing mutation(s) responsible for the drug inefficacy. It was then proposed that massive use of the drug would select for parasites harboring "genes" that confer resistance to the drug [75]. The presence of gene(s) that confer resistance to PZQ is not excluded, especially that a proportion of adult worms consistently survive treatment, and the drug is ineffective against immature worms. Yet, PZQ-resistant and susceptible worms did not show differences at 15 microsatellite genotypic markers [76].

Voltage-gated calcium channels that have been implicated in PZQ action did not show differences in the encoding cDNA sequence or expression levels among PZQ-susceptible and PZQ-resistant isolates [42-46, 77]. Adenosine triphosphate binding cassette (ABC) multi-drug transporters, especially the P glycoprotein, which shows selectivity for hydrophobic molecules, are predominantly localized in schistosome excretory system. Evidence for association between schistosome multi-drug transporters and PZQ resistance has been recently presented [78-81 and references therein].

On the other hand, Botros et al. [82] reported that laboratory-maintained S. mansoni isolates obtained from Egyptian patients resistant to PZQ cure failed to display PZQ-induced tegumental damage, implying changes in tegument characteristics in PZQ-unresponsive worms. Changes in parasite tegument properties in PZQ-resistant S. mansoni isolates were associated with decreased stability, reproductive fitness, and immunogenicity [65]. We recently proposed that the decrease in PZQ efficacy may be explained if repeated and intensive PZQ use selects for the worms with tighter outer lipid bilayer shield consequent to higher percentage of cholesterol and sphingomyelin (SM) and/or less active tegument-associated neutral sphingomyelinase (nSMase) [83]. The result would be worm progeny, able to prevent or decrease access of even molecules of $312 \mathrm{Da}$ such as PZQ [55]. Schistosomes showing PZQ insusceptibility were documented to incur serious biological costs [65, 78], a strong support to our assumption.

\subsection{Arachidonic acid}

\subsubsection{Structure, biological sources, and functions}

Arachidonic acid (ARA) is a polyunsaturated fatty acid, i.e., the 20 carbon-chain contains more (\#4) than one double bond, the first starting at position 6 from the last= omega carbon (methyl terminus opposite the $\mathrm{COOH}$ end). The formula is $\mathrm{C}_{20} \mathrm{H}_{32} \mathrm{O}_{2}, 20: 4(\omega-6)$. All double bonds are in a cis position (hydrogen atoms are on the same side of the double bonds) and, hence, ARA is also termed all-cis-5,8,11,14-eicosatetraenoic acid (Figure 2). ARA has an average mass of 304.467 Da and adopts a dominant hairpin conformation [84].

ARA is present throughout the body and comprises the greatest \% weight of total fatty acids among long chain poly-unsaturated fatty acids (LC-PUFAs). It is present as a structural component in animal and human cell membrane phospholipids, especially phosphatidylethanolamine, phosphatidylcholine, and phophatidylinositol. It is particularly abundant in skeletal muscles, the brain, and liver. ARA nutrition appears to serve various important roles throughout the human (and mammalian) life-cycle. Throughout pregnancy, ARA, along with 


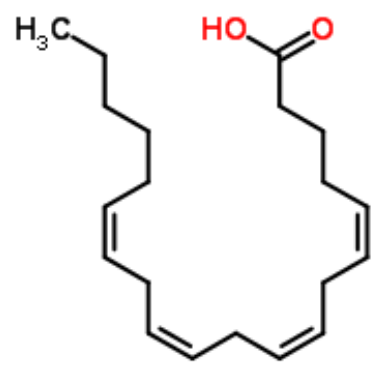

Figure 2. Structure of arachidonic acid.

other fats and nutrients, originates from the maternal diet and is supplied via the placenta to support the development of the growing fetus.

Between the third trimester of pregnancy and second year of neonatal life, the human brain undergoes a remarkable increase in the rate of growth. During this period, mass and volume increase more than 10 -fold, and achieve $>40 \%$ of the average-sized adult brain. These changes in size are mirrored by the accumulation of ARA in the brain [85, 86]. A major hallmark of mammalian birth is the sudden transition, following partuition, from the placenta as the source of prenatal nutrition, onto mother's milk as the main source from which to acquire nutrients. On average, ARA comprises $0.47 \pm 0.13 \%$ of total fatty acids in human milk [87]. The concentration of ARA in human milk varies between individuals and populations due, at least in part, to the presence of polymorphisms in the genes involved in the endogenous synthesis of LCPUFAs [88].

The amount of LC-PUFA intake by infants has been estimated based upon the assumptions that the fat content in human milk is $3.8 \mathrm{~g} / \mathrm{dL}$, and that average daily intake is $780 \mathrm{~mL} /$ day [89]. Using these values, average daily intake of ARA can be estimated to be approximately $140 \mathrm{mg}$. In addition, it is possible to compare the estimated average intake levels by infants to those of post-weaned individuals, due to the availability in the U.S. of annually collected data from dietary surveys [90].

The results from such a comparison reveal an interesting trend related to the levels of ARA consumed as individuals advance from pre- to post-weaning stages of human development. The average amount of dietary intake by toddler and preschool-age children (2-5 years) is approximately $80 \mathrm{mg}$. A modest increase in the absolute level of intake of approximately $20 \%$ occurs by school-age (6-11 years). Intake then decreases by adolescence (12-19 years), and once again approximates the average levels obtained through nursing. These differences in the amount of ARA consumed in the diet may initially seem to be marginal, until they are considered on a per weight basis. A change of approximately 20 -fold in body mass occurs as humans develop from infancy to adulthood (i.e., $\sim 3 \mathrm{~kg}$ to $\sim 60 \mathrm{~kg}$ ). Therefore, on a per weight basis, the amount of preformed ARA consumed daily by mid-adolescence is $\leq 5 \%$ relative to the estimated amount per $\mathrm{kg}$ of intake for infants. 
Although ARA is synthesized endogenously by the desaturation and elongation of linoleic acid (18:2n-6), results from experimental efforts consistently point to a need for ARA availability through dietary sources in order to elevate ARA in plasma and other tissues. In addition, associations between experimentally-defined endpoints and changes in the status of ARA reported in numerous investigations support the notion of a minimum required threshold in the concentrations of esterified and/or unesterified ARA in tissues or circulating pools [91].

The importance of preformed dietary ARA in supporting healthy immune functions has been demonstrated by investigations comparing the effects of supplementation using refined, microbial-derived ARA as triglycerides (TG) or unsupplemented formula and human milk on immune ability in pre-term and term infants. In an investigation involving infants born before 37 weeks of gestation, infants were provided with a control formula, or that same formula supplemented with $0.49 \%$ (wt/wt) of ARA, in addition to DHA [92-94]. After a period of 4 weeks, immunologic parameters were assessed from both groups and the results compared to those obtained from human milk-fed infants. The status of ARA in phospholipids was equivalent in the human milk-fed and LC-PUFA+formula-fed infants, and in both cases, significantly greater compared to infants fed with the control formula. In addition, effects on lymphocyte populations, cytokine production, and antibody maturation in formula-fed infants supplemented with LCPUFAs were consistent with those observed for human milkfed infants.

More recently, healthy full-term, formula-fed infants supplemented with $\leq 190 \mathrm{mg} /$ day ARA for a period of at least 8 weeks, showed fewer incidence of symptoms commonly associated with infections of the upper respiratory tract, in addition to fewer incidents of diarrhea. Importantly, the combined results from these investigations provide a clear example of the utility of preformed LC-PUFA in the diet to support the ability of infants to respond to immune challenges. In each case, the availability of preformed LC-PUFAs in the diet was associated with a preferred response to immune challenges, in contrast to results obtained from the use of formulations that contained adequate (i.e., 16\% wt/wt) 18:2n-6 [95].

Animal models have also been used to explore possible roles and functions of LC-PUFAs, including ARA, in immune-related activities. In piglets fed with formula enriched with supraphysiological levels of ARA resulted in maximal enrichment of intestinal mucosal within 8 days of initiating treatment. In addition, enrichment showed a dose-dependent response of intestinal mucosal phospholipid ARA concentration to dietary ARA in formula [96].

These results along with data from the human studies indicate that dietary intake of microbialderived ARA supports enrichment of ARA phospholipids in tissues. In addition, intake of sufficient levels of dietary ARA may support mucosal immunity against pathogenic agents, during periods of rapid growth and development in humans and other mammals. Overall, these and other results may not be surprising considering the large number of metabolic pathways and signaling cascades in which ARA is involved.

Arachidonic acid is essentially incorporated into cell phospholipids; in humans, approximately $10 \%$ of phopholipids in liver and plasma contain ARA [97-100]. N-arachidonoyl-phosphatidylethanol- amine leads via multiple pathways to anandamide, an endogenous cannabinoid neurotransmitter [101]. Arachidonic acid is liberated from phospholipids by the action of 
phospholipase A2, and then produces eicosanoids, prostaglandins, leukotrienes, and other essential bioactive products via the action of three types of oxygenases, cyclooxygenase (COX), lipoxygenase (LOX), and cytochrome P450. There is evidence that exogenous, plasma unesterified ARA may effectively compete with endogenous ARA for these enzymes, predominantly located on the nuclear and endoplasmic reticulum membrane. Some resultant eicosanoids are pro-inflammatory and vasoconstrictive, such as prostaglandin $E_{2}$, thromboxane $A_{2}$, and leukotriene $B_{4}$, and some are anti-inflammatory and anti-aggregatory such as prostacyclin and lipoxin $\mathrm{A}_{4}$ [97-100]. Indeed, shortly after inflammation is initiated, neutrophils that enter the tissue promote the switch of arachidonic acid-derived prostaglandins and leukotrienes to lipoxins, which induce resolution of the inflammation via termination of neutrophil recruitment and release of reparatory cytokines, such as transforming growth factor-beta [102].

\subsubsection{Dietary intake in humans}

In humans ARA is acquired directly from the diet or is derived by desaturation-elongation of linoleic acid (10-20 g daily) in the liver and in extra-hepatic tissues. ARA preference for acylation and transacylation reactions favors its partition in tissue phospholipids rather than adipose tissue and plasma triglycerides. Absorption of ingested ARA occurs in the endothelium of the intestinal lumen of the small intestine. Absorbed ARA is transported to various tissues and cells throughout the body by lipoproteins and acylated into sn-2 position of unesterified lysophospholipids, where it may remain until released by the enzymatic activation of phospholipase A2.

There is considerable evidence of the importance of ARA nutrition during early childhood development. ARA levels in circulation correlate positively with length to weight ratio and head circumference in neonates [91, 103]. In addition, the rate of ARA accretion by the brain increases exponentially during the brain growth-spurt, during the last trimester of pregnancy and into the first year of neonatal growth $[85,86]$. Tissue lipid data indicate that a $70 \mathrm{~kg}$ human contains 50-100 g ARA distributed between cell cytosolic and membrane compartments. It was estimated that on average, Australian diet provides ARA intake of $130 \mathrm{mg} /$ day for males and $96 \mathrm{mg} /$ day for females. Survey data for the intake of dietary ARA indicates a range of 80 to $190 \mathrm{mg} /$ day. Intake of ARA provided by a typical Japanese diet was calculated to be $300 \mathrm{mg} /$ day, respectively [104 and references therein]. To my knowledge, there is no information on the amounts of ARA diets in poor rural areas provide to children and pregnant women, but values are expectedly far less than those reported above. That is of concern as ARA is known to be essential for proper development of the brain and muscles in the fetus and in infants [91, 93, 105].

\subsubsection{Safety/adverse effects}

We recently conducted clinical trials on the safety of ARA intake in 20 and 90 Egyptian schoolage children of Menoufiya and Kafr El Sheikh Governorate, respectively. Arachidonic acid oral capsules, containing $396 \mathrm{mg}$ ARA (ARASCO®) per capsule, were provided by DSM (DSM Nutritional Products, Columbia, MD). Children were given $10 \mathrm{mg} / \mathrm{kg} /$ day (approximately 400 
mg daily) for 15 days over 3 weeks, i.e., 5 days per week. Not a single child reported the slighted malaise or adverse reaction during, and 6 weeks after, treatment. A panel of analyses indicated that biochemical profiles were either unchanged or ameliorated following ARA therapy in all children $[55,56]$. These results fully confirm the reports on safety of ARA in athletes supplemented with 1,000 mg for 50 days [106]. Regarding immunological analyses, intake of approximately $400 \mathrm{mg}$ ARA/day for 15 days led to significant $(P<0.0001)$ decrease of $S$. mansoni-infected Egyptian schoolchildren plasma levels of interleukin (IL)-10 and interferongamma (IFN- $\gamma$ ), thus rendering the plasma cytokine profile of the treated children nearer to normal $[55,56]$. Our findings are consistent with the observational studies showing that higher ARA intake was associated with lower levels of inflammatory markers and sequelae [107, 108].

The most serious adverse reaction reported for ARA was causing platelet aggregation in adults given $6 \mathrm{~g}$ ARA/day for 2 to 3 weeks [109]. However, approximately $0.5 \mathrm{~g}$ ARA daily intake for 15 days over 3 weeks did not alter platelet counts or blood coagulation parameters in $S$. mansoni-infected schoolchildren [55, 56], and healthy adults given $1.7 \mathrm{~g}$ ARA daily for 50 consecutive days [106]. In a double-blind, placebo-controlled study, supplementation of Japanese healthy adults with $838 \mathrm{mg} /$ day ARA for 2 weeks led to no changes in platelet aggregation [104].

\subsubsection{Schistosomicidal effects in vitro and in experimental animals}

The first indication on ARA schistosomicidal action was evident as we exposed ex vivo lungstage larvae of $S$. mansoni and $S$. haematobium to 0-500 $\mu \mathrm{M}$ ARA. Incubation in the presence of 10-20 $\mu \mathrm{M}$ ARA for 30 minutes induced exposure of otherwise hidden parasite surface membrane antigens to specific antibody binding. Higher ARA concentration or exposure time led to irreversible death of the larvae and destruction of their surface membrane. The monounsaturated omega-9 fatty acid, oleic acid (the major, $78.6 \%$, fatty acid in olive oil) alone or in conjunction with linoleic acid (as in corn oil), were able to expose the larval surface membrane antigens to antibody binding but did not induce attrition or membrane disruption. The study suggested that ARA is a schistosomicide for young larvae, with S. haematobium being more sensitive than S. mansoni [110]. Experiments with inhibitors and activators of neutral sphingomyelinase (nSMase) further indicated that ARA schistosomicidal effect is due, at least in part, to its ability to activate the parasite tegument-associated, magnesium-dependent nSMase [83].

Developing and adult S. mansoni and S. haematobium worms were also susceptible to in vitro schistosomicidal action whereby 2.5 and 5 mM ARA elicited irreversible killing of ex vivo 4-, 5-, and 6-week-old S. mansoni and 9-, 10-, and 12-week-old S. haematobium worms, within 3-4 hours, depending on the parasite age, even when worms were maintained in up to $50 \%$ fetal calf serum. Juvenile (3-week-old) S. mansoni were more sensitive to ARA as $100 \%$ irreversible attrition was noted 1 hour after exposure to 2.5 and $5 \mathrm{mM}$ concentration of ARA. Of note, ARAmediated worm attrition was prevented by concurrent incubation with nSMase inhibitors such as $\mathrm{CaCl}_{2}$ or $\mathrm{GW} 4869$, providing another evidence for the mechanism of ARA schistosomicidal action. Scanning and transmission electron microscopy revealed that ARA-mediated adult worm killing was associated with spine destruction, membrane blebbing, and disorganization of the apical membrane structure [111]. Expectedly, otherwise sequestered adult worm surface 
membrane antigens were accessible to specific antibody binding as judged by indirect membrane immunofluorescence. Additionally, addition of serum antibodies and peripheral blood mononuclear cells from patently infected hosts significantly enhanced ARA-mediated adult S. mansoni and S. haematobium attrition in vitro, suggesting that immune effectors strongly enhance ARA-mediated schistosomicidal effect [112].

Schistosomicidal activity of ARA was evident in vivo as a single oral dose of 500 and $1000 \mathrm{mg} /$ $\mathrm{kg}$ pure ARA administered to mice 7 and 35 days after $S$. mansoni infection, respectively elicited significant $(P<0.01)$ reduction of approximately $40 \%$ in worm burden, documenting the ARA susceptibility of larval and adult worms. Schistosoma haematobium appeared even more sensitive as a single oral dose of $1000 \mathrm{mg} / \mathrm{kg}$ ARA administered 70 days after infection induced highly significant $(P=0.003)$ reduction of $57.5 \%$ in worm burden evaluated 20 days later. Oral administration of mice with $300 \mathrm{mg} / \mathrm{kg}$ ARA incorporated in infant formula for 15 successive days led to even more highly significant $(P<0.0005)$ reduction of approximately $60 \%$ and $80 \%$ S. mansoni and S. haematobium worm burden, respectively [111]. These results were extended and entirely confirmed in hamster hosts where a series of 4 experiments for S. mansoni and S. haematobium indicated that ARA oral administration after patency led to highly significant $(P<0.02-<0.001)$ reduction in worm burden accompanied with significant $(P<0.05)$ decrease in worm egg load [112]. No adverse reactions were noted in any treated mouse or hamster. Additionally, no significant differences were noted among schistosome-infected and ARAtreated hamsters regarding serum total triglycerides and cholesterol levels, fatty acids relative percentages, erythrocytes and platelet count, and prothrombin and partial thromboplastin time, all evaluated in blood samples obtained 2 hours after ARA administration [112].

\subsubsection{Mechanism of schistosomicidal action}

We have previously shown that sphingomyelin (SM) in the schistosome apical lipid bilayer is the major responsible for the sieving properties of the outer membrane, which readily allows entry of nutrient molecules of $<600$ Da such as water, sugars, fatty acids, and cholesterol, while it prevents access of larger molecules, namely host antibodies (>150,000 Da), via interacting with surrounding water molecules to form a tight hydrogen barrier [83]. We have been able to use quasi-elastic neutron scattering to formally demonstrate the existence of the hydrogen barrier around schistosomes, and shown it is stronger in lung-stage larvae than adult worms and S. mansoni than S. haematobium. We have also shown that the hydrogen barrier collapses following nSMase activation and SM hydrolysis [113, 114].

We have predicted, and provided evidence for, the existence of schistosome tegumentassociated magnesium-dependent nSMase as early as 2006 [83]. The existence of the enzyme was formally demonstrated in 2009 following the analysis of S. mansoni complete genome [115]. The enzyme nucleotide and amino acid (aa) sequences were later fine-tuned [116]. We succeeded in cloning and sequencing $836 \mathrm{bp}$ near the 5' end of S. haematobium nSMaseencoding mRNA. The predicted amino acid sequences corresponded to aa18- aa277 in the $S$. mansoni counterpart with $96 \%$ identities and $98 \%$ positives, and contained the conserved domains characterizing the exonuclease-endonuclease-phosphatase (EEP) superfamily [12, 
117]. The complete S. haematobium genome and the complete sequence of the nSMase were published by Young et al. in 2012 [118].

We have been able to demonstrate the presence of nSMase in the tegument of adult $S$. mansoni and S. haematobium using enzymatic, immunologic, and immune-histochemical assays, and further shown that ARA was particularly potent in stimulating nSMase enzymatic activity, entirely supporting our previous findings using larvae and adult worms [12, 83, $110-114,117$, and references therein].

\subsubsection{Efficacy in school-age children}

In Menoufiya villages of low S. mansoni prevalence, efficacy of ARA in treatment of schoolage children with $<100$ epg was highly comparable to that of $\mathrm{PZQ}+$ Placebo (Pbo) with percent cure of $78 \%(11 / 14)$ and $85 \%(12 / 14)$, respectively, and similar levels of reduction in geometric mean egg counts (GMEC) in uncured patients. However, the efficacy of ARA in treatment of schoolchildren with 100-400 epg was significantly $(P<0.0001)$ lower than for PZQ regarding cure rate ( $44 \%$ and $83 \%$, respectively) and reduction in GMEC in uncured children, suggesting that ARA is indicated for treatment of light S. mansoni infection in children. In Kafr El Sheikh villages of high S. mansoni endemicity and where intensive mass PZQ treatment campaigns were implemented for 10 consecutive years, the efficacy of ARA in the treatment of school children with light infection ( $<100$ epg) was highly comparable to that of PZQ + Pbo with percent cure of $50 \%$ and $60 \%$, respectively. Efficacy of ARA and PZQ was again comparable in school-age children with heavy infection (800-1000 epg) leading to $21 \%$ and $20 \%$ cure rate, and $64 \%$ and $81 \%$ reduction in GMEC in uncured children, respectively. The results indicated that either PZQ or ARA are moderately efficacious for treatment of light S. mansoni infection in regions of elevated schistosomiasis prevalence, and neither should be used alone for treatment of children with heavy infections, especially those residing in regions of intense endemicity $[55,56]$.

It is important to assess the efficacy of ARA for treatment of S. haematobium. We expect high cure rates as larval and adult S. haematobium worms are shown in in vitro, and in vivo experiments in mice and hamsters to be more susceptible to ARA than S. mansoni [12, 83, 110-114-117].

\subsection{PZQ and arachidonic acid combination for treatment of human schistosomiasis}

\subsubsection{Evidence for safety and efficacy in children}

Intake of PZQ + ARA was as safe as for PZQ or ARA alone regarding metabolic panels or immunological parameters of S. mansoni-infected schoolchildren. Regarding efficacy, ARA and PZQ synergized for a spectacular cure of all lightly- and moderately-infected children residing in the low endemicity villages of Menoufiya, Egypt. In the high endemicity areas of Kafr El Sheikh, combining ARA and PZQ resulted into higher cure rates than mono-therapies for light, moderate, and heavy infection. For light infection, mono-therapy cure rate of 50-60\% was increased to $83 \%$ with the ARA + PZQ regimen. For the children with heavy infection, 
PZQ and ARA mono-therapy induced $20 \%$ cure rates, while cure rate of $78 \%$ was achieved with the drug combination $[55,56]$.

Combination of PZQ-oxamniquine (OXA) was assayed in randomized, non-blinded, doseranging trials in treatment of Malawi and Zimbabwe schoolchildren with low, moderate, and heavy infection. Cure rates were not available for Malawi children, while it reached $89 \%$ with the higher dose (20 and $10 \mathrm{mg} / \mathrm{kg}$ for PZQ and OXA, respectively) for children in Zimbabwe. No direct comparisons were reported for PZQ and OXA mono-therapy, and accordingly, synergistic effect was not documented [119-121]. Additionally, opposite to PZQ and ARA, OXA is effective against $S$. mansoni but not S. haematobium [121].

PZQ and artemether or artesunate in combination resulted in a protection rate of about $80 \%$, slightly higher than PZQ mono-therapy for treatment of schistosomiasis haematobium and japonicum in two field trials $[122,123]$. In a field trial in high S. mansoni endemicity Senegalese villages, 1 - to 60 -year-old (median=18 years) patients with moderate $S$. mansoni infection were treated with $\mathrm{PZQ}$, artesunate, or both drugs in combination (35-39 individuals per study arm). Cure rates were $44 \%$ for PZQ, $23 \%$ for artesunate, and $69 \%$ for the drugs combined. Combination of PZQ and artesunate appeared clearly synergistic, but the efficacy was obviously lower than for PZQ + ARA [123, 124]. This study revealed that efficacy of artemisinin derivative alone against $S$. mansoni is not evident and, furthermore, was not observed in S. haematobium infections [122]. Most importantly, artemether, and artemisin derivatives are used for malaria therapy; artemisinin resistance in Plasmodium falciparum is now prevalent across mainland Southeast Asia, and poses a threat to the control and elimination of malaria [125].

\subsubsection{Molecular basis for efficacy}

There were no reduction in efficacy of artemether and artesunate in killing PZQ-resistant and PZQ-susceptible S. japonicum worms in mice treated 7 and 8 or 35 and 36 days post-infection [126]. Opposite findings were observed regarding ARA, as its efficacy was as low as that of PZQ in treating schoolchildren with heavy S. mansoni infection, residing in areas where massive PZQ campaigns were applied for 10 consecutive years [55]. This finding implies that S. mansoni worms were resistant to mono-therapy with either PZQ or ARA. That has led us to propose that resistance to PZQ and ARA is attributed to continuous PZQ use, eliciting selection for worms with tight upper lipid bilayer, consequent to excessive SM synthesis and content or lower nSMase activity. Progeny of these worms would prevent access of even the 312 Da PZQ molecules, and would be less susceptible to ARA-mediated nSMase activation. Exposure of such worms to PZQ would not lead to their demise, yet likely facilitate ARA-mediated nSMase activation, SM hydrolysis and worm attrition. Exposure of these worms to ARA would lead to nSMase activation that is not lethal, yet sufficient to now allow entry of PZQ to perform its schistosomicidal action. Our proposition was supported by the considerable increase in cure rates in all children treated with PZQ + ARA versus the drugs' mono-therapy. The increase in cure rates was most highly significant $(P<0.0001)$ for children with heavy infections were cure rates with either PZQ or ARA alone were $20 \%$ and attained $78 \%$ with the drugs combined.

We shall test this hypothesis via evaluation of the cholesterol/SM content and nSMase activity in worms derived from cercariae obtained from communities of low prevalence/low PZQ use 
and high endemicity/intense PZQ administration. For documenting hypothesized mechanism for ARA counteraction of worm resistance to PZQ, larvae and worms that are progeny of entirely PZQ susceptible and strongly resistant breeds will be incubated with micromolar concentrations of ARA and then evaluated for responsiveness to PZQ, cholesterol/SM content, and nSMase activity.

\section{Conclusions and recommendations}

We may conclude that PZQ or ARA treatment shows considerable efficacy uniquely in the treatment of light infections in areas of low S. mansoni endemicity and PZQ use. Even then, efficacy never reaches $100 \%$, except when PZQ and ARA are combined. Chemotherapy with PZQ alone for patients residing in regions of high S. mansoni endemicity and afflicted with light, moderate, or heavy infection is not efficacious. Hence, it is recommended to implement optimal treatment regimens with PZQ in conjunction with ARA.

\section{Acknowledgements}

Experiments related to ARA efficacy and safety in mice and hamsters were supported by the Science and Technology Development Fund, Egypt, grants Nos. 144 and 2073 to R. El Ridi. For clinical trials in Menoufiya and Kafr El Sheikh, funding was provided by DSM North America, Columbia, Maryland.

\section{Author details}

Hatem Tallima ${ }^{1}$, Kevin Hadley ${ }^{2}$ and Rashika El Ridi ${ }^{*}$

*Address all correspondence to: rashikaelridi@hotmail.com

1 Zoology Department, Faculty of Science, Cairo University, Cairo, Egypt

2 Human Evidence Department, DSM North America, Columbia, Maryland, USA

\section{References}

[1] Barsoum RS, Esmat G, El-Baz T. Human schistosomiasis: Clinical perspective: Review. Journal of Advanced Research. 2013;4(5):433-444. 
[2] El Baz T, Esmat G. Hepatic and intestinal schistosomiasis: Review. Journal of Advanced Research. 2013;4(5):445-452.

[3] Barsoum RS. Urinary schistosomiasis: Review. Journal of Advanced Research. 2013;4(5):453-459.

[4] Bhardwaj R, Skelly PJ. Purinergic signaling and immune modulation at the schistosome surface? Trends in Parasitology. 2009;25(6):256-260.

[5] Othman A, El Ridi R. Schistosomiasis. In: Bruschi F. (ed.) Helminth Infections and Their Impact on Global Public Health. The Netherlands:Springer; 2014. p49-92.

[6] Chitsulo L, Loverde P, Engels D. Schistosomiasis. Nature Reviews Microbiology. 2004;2(1):12-13.

[7] WHO Technical Report Series 912: Prevention and control of schistosomiasis and soil-transmitted helminthiasis. Who Geneva, 2002 http://whqlibdoc.who.int/trs/ WHO_TRS_912.pdf.

[8] King CH. Parasites and poverty: The case of schistosomiasis. Acta Tropica. 2010;113(2):95-104.

[9] Hotez PJ, Alvarado M, Basáñez MG, Bolliger I, Bourne R, Boussinesq M, et al. The global burden of disease study 2010: Interpretation and implications for the neglected tropical diseases. PLoS Neglected Tropical Diseases. 2014;8(7):e2865.

[10] World Schistosomiasis Risk Chart. 2012 Edition. International Association for Medical Assistance to Travellers. www.IAMAT.Org.

[11] El Ridi RAF, Tallima HA-M. Novel therapeutic and prevention approaches for schistosomiasis: Review. Journal of Advanced Research. 2013;4(5):467-478.

[12] El Ridi R, Tallima H. Solving the riddle of the lung-stage schistosomula paved the way to a novel remedy and an efficacious vaccine for schistosomiasis. In: El Ridi R. (ed.) Parasitic Diseases - Schistosomiasis. Croatia: Intech Open; 2013. p179-202. www.intechopen.com/books/parasitic-diseases-schistosomiasis.

[13] Meyer F, Meyer H, Bueding E. Lipid metabolism in the parasitic and free-living flatworms, Schistosoma mansoni and Dugesia dorotocephala. Biochimica Biophysica Acta. 1970;210(2):257-266.

[14] Becker B, Mehlhorn H, Andrews P, Thomas H, Eckert J. Light and electron microscopic studies on the effect of praziquantel on Schistosoma mansoni, Dicrocoelium dendriticum, and Fasciola hepatica (Trematoda) in vitro. Zeitschrift fur Pflanzenernahrung und Bodenkunde. 1980;63(2):113-128.

[15] Mehlhorn H, Becker B, Andrews P, Thomas H, Frenkel JK. In vivo and in vitro experiments on the effects of praziquantel on Schistosoma mansoni. A light and electron microscopic study. Arzneimittel-Forschung. 1981;31(3a):544-554. 
[16] Sabah AA, Fletcher C, Webbe G, Doenhoff MJ. Schistosoma mansoni: Chemotherapy of infections of different ages. Experimental Parasitology. 1986;61(3):294-303.

[17] Shaw MK. Schistosoma mansoni: Stage-dependent damage after in vivo treatment with praziquantel. Parasitology. 1990;100(Pt 1):65-72.

[18] Flisser A, McLaren DJ. Effect of praziquantel treatment on lung-stage larvae of Schistosoma mansoni in vivo. Parasitology. 1989;98(Pt 2):203-211.

[19] Brindley PJ, Sher A. The chemotherapeutic effect of praziquantel against Schistosoma mansoni is dependent on host antibody response. Journal of Immunology. 1987;139(1):215-220.

[20] Brindley PJ, Sher A. Immunological involvement in the efficacy of praziquantel. Experimental Parasitolology. 1990;71(2):245-248.

[21] Doenhoff MJ, Modha J, Lambertucci JR, McLaren DJ. The immune dependence of chemotherapy. Parasitology Today. 1991;7(1):16-18.

[22] Fallon PG, Cooper RO, Probert AJ, Doenhoff MJ. Fallon PG, Fookes RE, Wharton GA. Temporal differences in praziquantel- and oxamniquine-induced tegumental damage to adult Schistosoma mansoni: implications for drug-antibody synergy. Parasitology. 1996;112(Pt 1):47-58.

[23] Webbe G, James C. A comparison of the susceptibility to praziquantel of Schistosoma haematobium, S. japonicum, S. mansoni, S. intercalatum and S. mattheei in hamsters. Zeitschrift fur Pflanzenernahrung und Bodenkunde. 1977;52(2):169-177.

[24] James C, Webbe G, Nelson GS. The susceptibility to praziquantel of Schistosoma haematobium in the baboon (Papio anubis) and of S. japonicum in the vervet monkey (Cercopithecus aethiops). Zeitschrift fur Pflanzenernahrung und Bodenkunde. 1977;52(2): 179-194.

[25] Andrews P. A summary of the efficacy of praziquantel against schistosomes in animal experiments and notes on its mode of action. Arzneimittel-Forschung. 1981;31(3a):538-541.

[26] Köhler P. The biochemical basis of anthelmintic action and resistance. International Journal for Parasitology. 2001;31(4): 336-345. Review.

[27] Harder A, Goossens J, Andrews P. Influence of praziquantel and Ca2+ on the bilayerisotropic-hexagonal transition of model membranes. Molecular and Biochemical Parasitology. 1988;29(1):55-59.

[28] Schepers H, Brasseur R, Goormaghtigh E, Duquenoy P, Ruysschaert JM. Mode of insertion of praziquantel and derivatives into lipid membranes. Biochemical Pharmacolology. 1988;37(8):1615-16 23. 
[29] Lima SF, Vieira LQ, Harder A, Kusel JR. Altered behaviour of carbohydrate-bound molecules and lipids in areas of the tegument of adult Schistosoma mansoni worms damaged by praziquantel. Parasitology. 1994;100(Pt 4):469-477.

[30] Kusumi A, Nakada C, Ritchie K, Murase K, Suzuki K, Murakoshi H, Kasai RS, Kondo J, Fujiwara T. Paradigm shift of the plasma membrane concept from the two-dimensional continuum fluid to the partitioned fluid: high-speed single-molecule tracking of membrane molecules. Annual Review of Biophysics and Biomolecular Structure. 2005;34:351-378. Review.

[31] Cohen C, Reinhardt B, Castellani L, Norton P, Stirewalt M. Schistosome surface spines are "crystals" of actin. The journal of Cell biology. 1982;95(3):987-988.

[32] Zhou Y, Podesta RB. Surface spines of human blood flukes (Schistosoma mansoni) contain bundles of actin filaments having identical polarity. European Journal of Cell Biology. 1989Feb;48(1):150-153.

[33] Abbas MK, Cain GD. Actin and intermediate-sized filaments of the spines and cytoskeleton of Schistosoma mansoni. Parasitological Research. 1987;73(1):66-74.

[34] Viola A, Gupta N. Tether and trap: regulation of membrane-raft dynamics by actinbinding proteins. Nature Reviews Immunology. 2007;7(11):889-896. Review.

[35] Morone N. Freeze-etch electron tomography for the plasma membrane interface. Methods in Molecular Biology. 2010;657:275-86.

[36] Bricker CS, Depenbusch JW, Bennett JL, Thompson DP. The relationship between tegumental disruption and muscle contraction in Schistosoma mansoni exposed to various compounds. Zeitschrift fur Pflanzenernahrung und Bodenkunde. 1983;69(1): 61-71.

[37] Trivedi DV, Muretta JM, Swenson AM, Thomas DD, Yengo CM. Magnesium impacts myosin $\mathrm{V}$ motor activity by altering key conformational changes in the mechanochemical cycle. Biochemistry. 2013;52(27):4710-4722.

[38] Tallima H, El Ridi R. Praziquantel binds Schistosoma mansoni adult worm actin. International Journal of Antimicrobial Agents. 2007;29(5):570-575.

[39] Tallima H, El Ridi R. Re: Is actin the praziquantel receptor? International Journal of Antimicrobial Agents. 2007;30(6):566-567.

[40] Pica-Mattoccia L, Valle C, Basso A, Troiani AR, Vigorosi F, Liberti P, Festucci A, Cioli D. Cytochalasin D abolishes the schistosomicidal activity of praziquantel. Experimental Parasitology. 2007;115(4):344-351.

[41] Gnanasekar M, Salunkhe AM, Mallia AK, He YX, Kalyanasundaram R. Praziquantel affects the regulatory myosin light chain of Schistosoma mansoni. Antimicrobial Agents and Chemotherapy 2009;53(3):1054-1060.

[42] Kohn AB, Anderson PA, Roberts-Misterly JM, Greenberg RM. Schistosome calcium channel beta subunits. Unusual modulatory effects and potential role in the action of 
the antischistosomal drug praziquantel. Journal of Biological Chemistry. 2001;276(40):36873-36876.

[43] Kohn AB, Lea J, Roberts-Misterly JM, Anderson PA, Greenberg RM. Structure of three high voltage-activated calcium channel alpha1 subunits from Schistosoma mansoni. Parasitology. 2001;123(Pt 5):489-497.

[44] Kohn AB, Roberts-Misterly JM, Anderson PA, Khan N, Greenberg RM. Specific sites in the Beta Interaction Domain of a schistosome Ca2+ channel beta subunit are key to its role in sensitivity to the anti-schistosomal drug praziquantel. Parasitology. 2003;127(Pt 4):349-356.

[45] Kohn AB, Roberts-Misterly JM, Anderson PA, Greenberg RM. Creation by mutagenesis of a mammalian $\mathrm{Ca}(2+)$ channel beta subunit that confers praziquantel sensitivity to a mammalian $\mathrm{Ca}(2+)$ channel. International Journal for Parasitology. 2003;33(12):1303-1308.

[46] Salvador-Recatalà V, Greenberg RM. Calcium channels of schistosomes: unresolved questions and unexpected answers. Wiley Interdisciplinary Review of Membrane Transport and Signaling. 2012;1(1):85-93.

[47] Pica-Mattoccia L, Orsini T, Basso A, Festucci A, Liberti P, Guidi A, Marcatto-Maggi AL, Nobre-Santana S, Troiani AR, Cioli D, Valle C. Schistosoma mansoni: lack of correlation between praziquantel-induced intra-worm calcium influx and parasite death. Experimental Parasitology. 2008;119(3):332-335.

[48] Olliaro PL, Vaillant MT, Belizario VJ, Lwambo NJ, Ouldabdallahi M, Pieri OS, Amarillo ML, Kaatano GM, Diaw M, Domingues AC, Favre TC, Lapujade O, Alves F, Chitsulo L. A multicentre randomized controlled trial of the efficacy and safety of single-dose praziquantel at $40 \mathrm{mg} / \mathrm{kg}$ vs. $60 \mathrm{mg} / \mathrm{kg}$ for treating intestinal schistosomiasis in the Philippines, Mauritania, Tanzania and Brazil. PLoS Neglected Tropical Diseases. 2011;5(6):e1165.

[49] Zwang J, Olliaro PL. Clinical efficacy and tolerability of praziquantel for intestinal and urinary schistosomiasis-a meta-analysis of comparative and non-comparative clinical trials. PLoS Neglected Tropical Diseases. 2014;8(11):e3286.

[50] Barakat R, El Morshedy H. Efficacy of two praziquantel treatments among primary school children in an area of high Schistosoma mansoni endemicity, Nile Delta, Egypt. Parasitology. 2011;138(4):440-446.

[51] Berhe N, Gundersen SG, Abebe F, Birrie H, Medhin G, Gemetchu T. Praziquantel side effects and efficacy related to Schistosoma mansoni egg loads and morbidity in primary school children in north-east Ethiopia. Acta Tropica. 1999;72(1):53-63.

[52] Utzinger J, N'Goran EK, N'Dri A, Lengeler C, Tanner M. Efficacy of praziquantel against Schistosoma mansoni with particular consideration for intensity of infection. Tropical Medicine and International Health. 2000;5(11):771-778. 
[53] Midzi N, Sangweme D, Zinyowera S, Mapingure MP, Brouwer KC, Kumar N, Mutapi F, Woelk G, Mduluza T. Efficacy and side effects of praziquantel treatment against Schistosoma haematobium infection among primary school children in Zimbabwe. Transactions of the Royal Society of Tropical Medicine and Hygiene. 2008;102(8): 759-766.

[54] King CH, Olbrych SK, Soon M, Singer ME, Carter J, Colley DG. Utility of repeated praziquantel dosing in the treatment of schistosomiasis in high-risk communities in Africa: A systematic review. PLoS Neglected Tropical Diseases. 2011;5(9):e1321.

[55] Barakat R, Abou El-Ela N, Sharaf S, El Sagheer O, Selim S, Tallima H, Bruins MJ, Hadley KB, El Ridi R. Efficacy and safety of arachidonic acid for treatment of schoolage children in Schistosoma mansoni high endemicity regions. American Journal of Tropical Medicine and Hygiene. 2015;92(4):797-894.

[56] Selim S, El Sagheer O, El Amir A, Barakat R, Hadley K, Bruins MJ, El Ridi R. Efficacy and safety of arachidonic acid for treatment of Schistosoma mansoni-infected children in Menoufiya, Egypt. American Journal of Tropical Medicine and Hygiene. 2014;91(5):973-981.

[57] Tchuem Tchuenté LA, Momo SC, Stothard JR, Rollinson D. Efficacy of praziquantel and reinfection patterns in single and mixed infection foci for intestinal and urogenital schistosomiasis in Cameroon. Acta Tropica. 2013;128(2):275-283.

[58] Reta B, Erko B. Efficacy and side effects of praziquantel in the treatment for Schistosoma mansoni infection in school children in Senbete Town, northeastern Ethiopia. Tropical Medicine and International Health. 2013;18(11):1338-1343.

[59] Erko B, Degarege A, Tadesse K, Mathiwos A, Legesse M, 2012. Efficacy and side effects of praziquantel in the treatment of Schistosomiasis mansoni in schoolchildren in Shesha Kekele Elementary School, Wondo Genet, Southern Ethiopia. Asian Pacific Journal of Tropical Biomedicine. 2012;2(3):235-239.

[60] Black CL, Steinauer ML, Mwinzi PN, Evan Secor W, Karanja DM, Colley DG. Impact of intense, longitudinal retreatment with praziquantel on cure rates of schistosomiasis mansoni in a cohort of occupationally exposed adults in western Kenya. Tropical Medicine and International Health. 2009;14:450-457.

[61] Picquet M, Vercruysse J, Shaw DJ, Diop M, Ly A. Efficacy of praziquantel against Schistosoma mansoni in northern Senegal. Transactions of the Royal Society of Tropical Medicine and Hygiene. 1998;92(1):90-93.

[62] Gryseels B, Mbaye A, De Vlas SJ, Stelma FF, Guissé F, Van Lieshout L, Faye D, Diop M, Ly A, Tchuem-Tchuenté LA, Engels D, Polman K. Are poor responses to praziquantel for the treatment of Schistosoma mansoni infections in Senegal due to resistance? An overview of the evidence. Tropical Medicine and International Health. 2001;6(11):864-873. 
[63] Webster BL, Diaw OT, Seye MM, Faye DS, Stothard JR, Sousa-Figueiredo JC, Rollinson D, 2013. Praziquantel treatment of school children from single and mixed infection foci of intestinal and urogenital schistosomiasis along the Senegal River Basin: monitoring treatment success and re-infection patterns. Acta Tropica. 2013;128(2): 292-302.

[64] Ismail M, Botros S, Metwally A, William S, Farghally A, Tao LF, Day TA, Bennett JL. Resistance to praziquantel: Direct evidence from Schistosoma mansoni isolated from Egyptian villagers. American Journal of Tropical Medicine and Hygiene. 1999;60(6): 932-935.

[65] [65]William S, Sabra A, Ramzy F, Mousa M, Demerdash Z, Bennett JL, Day TA, Botros S. Stability and reproductive fitness of Schistosoma mansoni isolates with decreased sensitivity to praziquantel. International Journal for Parasitology. 2001;31(10):1093-1100.

[66] Doenhoff MJ, Kusel JR, Coles GC, Cioli D. Resistance of Schistosoma mansoni to praziquantel: Is there a problem? Transactions of the Royal Society of Tropical Medicine and Hygiene. 2002;96(5):465-469.

[67] Botros S, Sayed H, Amer N, El-Ghannam M, Bennett JL, Day TA. Current status of sensitivity to praziquantel in a focus of potential drug resistance in Egypt. International Journal for Parasitolology. 2005;35(7):787-791.

[68] Lawn SD1, Lucas SB, Chiodini PL. Case report: Schistosoma mansoni infection: failure of standard treatment with praziquantel in a returned traveller. Transactions of the Royal Society of Tropical Medicine and Hygiene. 2003;97(1):100-101.

[69] Wang W, Wang L, Liang YS. Susceptibility or resistance of praziquantel in human schistosomiasis: A review. Parasitological Research. 2012;111(5):1871-1877.

[70] Melman SD, Steinauer ML, Cunningham C, Kubatko LS, Mwangi IN, Wynn NB, Mutuku MW, Karanja DM, Colley DG, Black CL, Secor WE, Mkoji GM, Loker ES. Reduced susceptibility to praziquantel among naturally occurring Kenyan isolates of Schistosoma mansoni. PLoS Neglected Tropical Diseases. 2009;3(8):e504.

[71] Mwangi IN, Sanchez MC, Mkoji GM, Agola LE, Runo SM, Cupit PM, Cunningham C. Praziquantel sensitivity of Kenyan Schistosoma mansoni isolates and the generation of a laboratory strain with reduced susceptibility to the drug. International Journal for Parasitology: Drugs and Drug Resistance. 2014;4(3):296-300.

[72] Cioli D, Pica-Mattoccia L, Archer S. Drug resistance in schistosomes. Parasitology Today. 1993;9(5):162-166.

[73] Pica-Mattoccia L, Dias LC, Moroni R, Cioli D. Schistosoma mansoni: Genetic complementation analysis shows that two independent hycanthone/oxamniquine-resistant strains are mutated in the same gene. Experimental Parasitology. 1993;77(4):445-449.

[74] Valentim CL, Cioli D, Chevalier FD, Cao X, Taylor AB, Holloway SP, Pica-Mattoccia L, Guidi A, Basso A, Tsai IJ, Berriman M, Carvalho-Queiroz C, Almeida M, Aguilar 
H, Frantz DE, Hart PJ, LoVerde PT, Anderson TJ. Genetic and molecular basis of drug resistance and species-specific drug action in schistosome parasites. Science. 2013;342(6164):1385-1389.

[75] Fallon PG, Tao LF, Ismail MM, Bennett JL. Schistosome resistance to praziquantel: Fact or artifact? Parasitology Today. 1996;12(8):316-320.

[76] Blanton RE1, Blank WA, Costa JM, Carmo TM, Reis EA, Silva LK, Barbosa LM, Test MR, Reis MG. Schistosoma mansoni population structure and persistence after praziquantel treatment in two villages of Bahia, Brazil. International Journal for Parasitology. 2011;41(10):1093-1099.

[77] Valle C, Troiani AR, Festucci A, Pica-Mattoccia L, Liberti P, Wolstenholme A, Francklow K, Doenhoff MJ, Cioli D. Sequence and level of endogenous expression of calcium channel beta subunits in Schistosoma mansoni displaying different susceptibilities to praziquantel. Molecular and Biochemical Parasitology. 2003 31;130(2):111-115.

[78] [78]Greenberg RM. New approaches for understanding mechanisms of drug resistance in schistosomes. Parasitology. 2013;140(12):1534-1546.

[79] Kasinathan RS, Sharma LK, Cunningham C, Webb TR, Greenberg RM. Inhibition or knockdown of $A B C$ transporters enhances susceptibility of adult and juvenile schistosomes to Praziquantel. PLoS Neglected Tropical Diseases. 2014;8(10):e3265

[80] Greenberg RM. Schistosome ABC multidrug transporters: From pharmacology to physiology. International Journal for Parasitology: Drugs and Drug Resistance. 2014;4(3):301-309.

[81] Greenberg RM. Ion channels and drug transporters as targets for anthelmintics. Current Clinical Microbiology Reports. 2014;1(3-4):51-60.

[82] William S, Botros S, Ismail M, Farghally A, Day TA, Bennett JL. Praziquantel-induced tegumental damage in vitro is diminished in schistosomes derived from praziquantel-resistant infections. Parasitology. 2001;122(Pt 1):63-66.

[83] El Ridi R, Tallima H. Equilibrium in lung schistosomula sphingomyelin breakdown and biosynthesis allows very small molecules, but not antibody, to access proteins at the host-parasite interface. Journal of Parasitology. 2006;92(4):730-737.

[84] Rich MR. Conformational analysis of arachidonic and related fatty acids using molecular dynamics simulations. Biochimica Biophysica Acta. 1993;1178(1):87-96.

[85] Martinez M. (1992). Tissue levels of polyunsaturated fatty acids during early human development. The Journal of Pediatrics. 1992;120(4):S129-S138.

[86] Carlson SE, Werkman SH, Peeples JM, Wilson WM 3rd. Growth and development of premature infants in relation to omega 3 and omega 6 fatty acid status. World Review of Nutrition and Dietetics 1994;75:63-69. 
[87] Brenna JT, Varamini B, Jensen RG, Diersen-Schade DA, Boettcher JA, Arterburn LM. Docosahexaenoic and arachidonic acid concentrations in human breast milk worldwide. The American Journal of Clinical Nutrition. 2007;85(6):1457-1464.

[88] Scholtz SA, Kerling EH, Shaddy DJ, Li S, Thodosoff JM, Colombo J, Carlson SE (2014). Docosahexaenoic acid (DHA) supplementation in pregnancy differentially modulates arachidonic acid and DHA status across FADS genotypes in pregnancy. Prostaglandins, Leukotrienes and Essential Fatty Acids (PLEFA). 2014; pii: S0952-3278(14)00186-0. doi: 10.1016/j.plefa.2014.10.008, in press.

[89] Innis SM. Fatty acids and early human development. Early human development. 2007;83(12):761-766.

[90] U.S. Department of Agriculture, Agricultural Research Service, Beltsville Human Nutrition Research Center, Food Surveys Research Group (Beltsville, MD) and U.S. Department of Health and Human Services, Centers for Disease Control and Prevention, National Center for Health Statistics (Hyattsville, MD). What We Eat in America, NHANES 2009-2010 Data: Dietary Interview - Total Nutrients Intakes -- First Day (DR1TOT_C). (2009, September). Available from: http://www.ars.usda.gov/SP2UserFiles/Place/80400530/pdf/0910/Table_1_NIN_GEN_09.pdf [accessed 1/22/15].

[91] Carlson SE, Werkman SH, Peeples JM, Cooke R J, Tolley EA. Arachidonic acid status correlates with first year growth in preterm infants. Proceedings of the National Academy of Sciences USA. 1993;90(3):1073-1077.

[92] Field CJ, Thomson CA, Van Aerde JE, Parrott A, Lien E, Clandinin MT. Lower proportion of CD45R0+ cells and deficient interleukin-10 production by formula-fed infants, compared with human-fed, is corrected with supplementation of long-chain polyunsaturated fatty acids. Journal of Pediatric Gastroenterology and Nutrition. 2000;31(3):291-299.

[93] Birch EE, Garfield S, Hoffman DR, Uauy R, Birch DG. A randomized controlled trial of early dietary supply of long-chain polyunsaturated fatty acids and mental development in term infants. Developmental Medicine and Child Neurology. 2000;42(3): 174-181.

[94] Lapillonne A, Pastor N, Zhuang W, Scalabrin DM. Infants fed formula with added long chain polyunsaturated fatty acids have reduced incidence of respiratory illnesses and diarrhea during the first year of life. BMC Pediatrics. 2014:14(1):168.

[95] Field CJ, Van Aerde JE, Robinson LE, Thomas Clandinin M. Effect of providing a formula supplemented with long-chain polyunsaturated fatty acids on immunity in full-term neonates. British Journal of Nutrition. 2008;99(1):91-99.

[96] Hess HA, Corl BA, Lin X, Jacobi SK, Harrell RJ, Blikslager AT, Odle J. Enrichment of intestinal mucosal phospholipids with arachidonic and eicosapentaenoic acids fed to suckling piglets is dose and time dependent. The Journal of Nutrition. 2008;138(11): 2164-2171. 
[97] Thomas JM, Hullin F, Chap H, Douste-Blazy L. Phosphatidylcholine is the major phospholipid providing arachidonic acid for prostacyclin synthesis in thrombinstimulated human endothelial cells. Thrombosis Research. 1984;34(2):117-123.

[98] Zhou L, Vessby B, Nilsson A. Quantitative role of plasma free fatty acids in the supply of arachidonic acid to extrahepatic tissues in rats. Journal of Nutrition. 2002;132(9):2626-2631.

[99] Brash AR. Arachidonic acid as a bioactive molecule. The Journal of Clinical Investigation. 2001;107(11):1339-1345.

[100] Zhou L, Nilsson A. Sources of eicosanoid precursor fatty acid pools in tissues. Journal of Lipid Research. 2001;42(10):1521-42. Review.

[101] Wang J, Ueda N. Biology of endocannabinoid synthesis system. Prostaglandins and Other Lipid Mediators. 2009;89(3-4):112-129.

[102] Serhan CN, Savill J. Resolution of inflammation: The beginning programs the end. Nature Immunology. 2005;6(12):1191-1197.

[103] Leaf AA, Leighfield MJ, Costeloe KL, Crawford MA. Long chain polyunsaturated fatty acids and fetal growth. Early Human Development. 1992;30(3):183-191.

[104] Kusumoto A, Ishikura Y, Kawashima H, Kiso Y, Takai S, Miyazaki M. Effects of arachidonate-enriched triacylglycerol supplementation on serum fatty acids and platelet aggregation in healthy male subjects with a fish diet. British Journal of Nutrition. 2007;98(3):626-635.

[105] Kohn G, Sawatzki G, van Biervliet JP, Rosseneu M. Diet and the essential fatty acid status of term infants. Acta Paediatrica Supplement. 1994;402:69-74.

[106] Roberts MD, Iosia M, Kerksick CM, Taylor LW, Campbell B, Wilborn CD, Harvey T, Cooke M, Rasmussen C, Greenwood M, Wilson R, Jitomir J, Willoughby D, Kreider RB. Effects of arachidonic acid supplementation on training adaptations in resistance-trained males. Journal of the International Society of Sports Nutrition. 2007;4:21.

[107] Ferrucci L, Cherubini A, Bandinelli S, Bartali B, Corsi A, Lauretani F, Martin A, Andres-Lacueva C, Senin U, Guralnik JM. Relationship of plasma polyunsaturated fatty acids to circulating inflammatory markers. The Journal of Clinical Endocrinology and Metabolism. 2006Feb;91(2):439-446.

[108] Harris WS, Mozaffarian D, Rimm E, Kris-Etherton P, Rudel LL, Appel LJ, Engler MM, Engler MB, Sacks F. Omega- 6 fatty acids and risk for cardiovascular disease: a science advisory from the American Heart Association Nutrition Subcommittee of the Council on Nutrition, Physical Activity, and Metabolism; Council on Cardiovascular Nursing; and Council on Epidemiology and Prevention. Circulation. 2009;119(6):902-907.

[109] Seyberth HW, Oelz O, Kennedy T, Sweetman BJ, Danon A, Frölich JC, Heimberg M, Oates JA. Increased arachidonate in lipids after administration to man: effects on 
prostaglandin biosynthesis. Clinical Pharmacology and Therapeutics. 1975;18(5 Pt 1): 521-529.

[110] Tallima H, Salah M, El-Ridi R. In vitro and in vivo effects of unsaturated fatty acids on Schistosoma mansoni and S. haematobium lung-stage larvae. Journal of Parasitology. 2005;91(5):1094-1102.

[111] El Ridi R, Aboueldahab M, Tallima H, Salah M, Mahana N, Fawzi S, Mohamed SH, Fahmy OM. In vitro and in vivo activities of arachidonic acid against Schistosoma mansoni and Schistosoma haematobium. Antimicrobial Agents and Chemotherapy. 2010;54(8):3383-3389.

[112] El Ridi R, Tallima H, Salah M, Aboueldahab M, Fahmy OM, Al-Halbosiy MF, Mahmoud SS. Efficacy and mechanism of action of arachidonic acid in the treatment of hamsters infected with Schistosoma mansoni or Schistosoma haematobium. International Journal of Antimicrobial Agents. 2012;39(3):232-239.

[113] Migliardo F, Tallima H, El Ridi R. Is there a sphingomyelin-based hydrogen bond barrier at the mammalian host-schistosome parasite interface? Cell Biochemistry and Biophysics. 2014;68(2):359-367.

[114] Migliardo F, Tallima H, El Ridi R. Rigidity and resistance of larval- and adult schistosomes-medium interface. Biochemical and Biophysical Research Communications. 2014;446(1):255-260.

[115] Berriman M, Haas BJ, LoVerde PT, Wilson RA, Dillon GP, Cerqueira GC, Mashiyama ST, Al-Lazikani B, Andrade LF, Ashton PD, Aslett MA, Bartholomeu DC, Blandin G, Caffrey CR, Coghlan A, Coulson R, Day TA, Delcher A, DeMarco R, Djikeng A, Eyre T, Gamble JA, Ghedin E, Gu Y, Hertz-Fowler C, Hirai H, Hirai Y, Houston R, Ivens A, Johnston DA, Lacerda D, Macedo CD, McVeigh P, Ning Z, Oliveira G, Overington JP, Parkhill J, Pertea M, Pierce RJ, Protasio AV, Quail MA, Rajandream MA, Rogers J, Sajid M, Salzberg SL, Stanke M, Tivey AR, White O, Williams DL, Wortman J, Wu W, Zamanian M, Zerlotini A, Fraser-Liggett CM, Barrell BG, El-Sayed NM. The genome of the blood fluke Schistosoma mansoni. Nature. 2009;460(7253):352-358.

[116] Protasio AV, Tsai IJ, Babbage A, Nichol S, Hunt M, Aslett MA, De Silva N, Velarde GS, Anderson TJ, Clark RC, Davidson C, Dillon GP, Holroyd NE, LoVerde PT, Lloyd C, McQuillan J, Oliveira G, Otto TD, Parker-Manuel SJ, Quail MA, Wilson RA, Zerlotini A, Dunne DW, Berriman M. A systematically improved high quality genome and transcriptome of the human blood fluke Schistosoma mansoni. PLoS Neglected Tropical Diseases. 2012;6(1):e1455.

[117] Tallima H, Al-Halbosiy MF, El Ridi R. Enzymatic activity and immunolocalization of Schistosoma mansoni and Schistosoma haematobium neutral sphingomyelinase. Molecular and Biochemical Parasitology. 2011;178(1-2):23-28.

[118] Young ND, Jex AR, Li B, Liu S, Yang L, Xiong Z, Li Y, Cantacessi C, Hall RS, Xu X, Chen F, Wu X, Zerlotini A, Oliveira G, Hofmann A, Zhang G, Fang X, Kang Y, 
Campbell BE, Loukas A, Ranganathan S, Rollinson D, Rinaldi G, Brindley PJ, Yang $\mathrm{H}$, Wang J, Wang J, Gasser RB. Whole-genome sequence of Schistosoma haematobium. Nature Genetics. 2012;44(2):221-225.

[119] Pugh RN, Teesdale CH. Synergy of concurrent low dose oxamniquine and praziquantel in schistosomiasis. British Medical Journal (Clinical Research Ed). 1983;287(6396): 877-878.

[120] Creasey AM, Taylor P, Thomas JE. Dosage trial of a combination of oxamniquine and praziquantel in the treatment of schistosomiasis in Zimbabwean schoolchildren. Central African Journal of Medicine. 1986;32(7):165-167.

[121] Utzinger J, Keiser J, Shuhua X, Tanner M, Singer BH. Combination chemotherapy of schistosomiasis in laboratory studies and clinical trials. Antimicrobial Agents and Chemotherapy. 2003;47(5):1487-1495.

[122] Borrmann S, Szlezák N, Faucher JF, Matsiegui PB, Neubauer R, Binder RK, Lell B, Kremsner PG. Artesunate and praziquantel for the treatment of Schistosoma haematobium infections: A double-blind, randomized, placebo-controlled study. Journal of Infectious Diseases. 2001;184(10):1363-1366.

[123] Liu R, Dong HF, Guo Y, Zhao QP, Jiang MS. Efficacy of praziquantel and artemisinin derivatives for the treatment and prevention of human schistosomiasis: A systematic review and meta-analysis. Parasites and Vectors. 2011;4:201.

[124] De Clercq D, Vercruysse J, Verlé P, Kongs A, Diop M. What is the effect of combining artesunate and praziquantel in the treatment of Schistosoma mansoni infections? Tropical Medicine and International Health. 2000;5(10):744-746.

[125] Ashley EA, Dhorda M, Fairhurst RM, Amaratunga C, Lim P, Suon S, et al. Spread of artemisinin resistance in Plasmodium falciparum malaria. New England Journal of Medicine. 2014;371(5):411-423.

[126] Wang W, Li TY, Ji Y, Qu GL, Qian YL, Li HJ, Dai JR, Liang YS. Efficacy of artemether and artesunate in mice infected with praziquantel non-susceptible isolate of Schistosoma japonicum. Parasitology Research. 2014;113(3):925-931. 